\title{
Cost-effectiveness of using continuous positive airway pressure in the treatment of severe obstructive sleep apnoea/hypopnoea syndrome in the UK
}

\author{
J F Guest, ${ }^{1,2}$ M T Helter, ${ }^{1}$ A Morga, ${ }^{1}$ J R Stradling ${ }^{3}$
}

${ }^{1}$ Catalyst Health Economics Consultants, Northwood, Middlesex, UK; ${ }^{2}$ Postgraduate Medical School, University of Surrey, Guildford, Surrey, UK;

${ }^{3}$ Respiratory Sleep Unit, Churchill Hospital, Oxford, UK

Correspondence to: Professor J F Guest, Catalyst Health Economics Consultants, 34b High Street, Northwood, Middlesex HA6 1BN, UK; julian. guest@catalyst-health.co.uk

Received 30 June 2007 Accepted 16 March 2008 Published Online First 11 April 2008

\begin{abstract}
Objective: A study was undertaken to estimate the costeffectiveness of using continuous positive airway pressure (CPAP) in the management of patients with severe obstructive sleep apnoea/hypopnoea syndrome (OSAHS) compared with no treatment from the perspective of the UK's National Health Service (NHS).
\end{abstract}

Methods: A Markov model was constructed to assess the cost-effectiveness of CPAP compared with no treatment. The model depicted the management of a 55year-old patient with severe OSAHS as defined by an apnoea-hypopnoea index (AHI) $>30$ and daytime sleepiness (Epworth Sleepiness Scale score $\geqslant 12$ ). The model spans a period of 14 years.

Results: According to the model, $57 \%$ of untreated patients are expected to be alive at the end of 14 years compared with $72 \%$ of patients treated with CPAP. Untreated patients are expected to cost the NHS f10 645 (95\% Cl f7988 to $£ 14$ 098) per patient over 14 years compared with $£ 9672$ (95\% Cl $£ 8057$ to $£ 12$ 860) per CPAP-treated patient. Treatment with CPAP for a period of 1 year was found not to be a cost-effective option since the cost per quality-adjusted life year (QALY) gained is expected to be $>£ 20000$, but after 2 years of treatment the cost per QALY gained is expected to be f10 000 or less and, after 13 years of treatment, CPAP becomes a dominant treatment (ie, more effective than no treatment for less cost).

Conclusion: Within the limitations of the model, CPAP was found to be clinically more effective than no treatment and, from the perspective of the UK's NHS, a cost-effective strategy after a minimum of 2 years of treatment.

The nocturnal severity of obstructive sleep apnoea/ hypopnoea syndrome (OSAHS) is often defined by the number of events per hour. This could be either the number of oxygen desaturation events (oxygen desaturation index) or the number of apnoeas and hypopnoeas (apnoea hypopnoea index (AHI)), with arbitrary thresholds to define mild, moderate and severe disease. The daytime symptom severity of OSAHS is often defined by either subjective sleepiness scales (such as the Epworth Sleepiness Scale (ESS)) or by objective tests of propensity to sleep (such as the maintenance of wakefulness test).

Continuous positive airway pressure (CPAP) therapy is the treatment of choice for patients with moderate to severe OSAHS, established through meta-analyses. ${ }^{12}$ In most cases the alternative for patients who cannot tolerate CPAP is no treatment. Against this background, this study estimated the cost-effectiveness of using CPAP in the management of patients with severe OSAHS compared with no treatment in the UK.

\section{METHODS}

\section{Perspective}

This analysis estimated the direct healthcare costs and benefits of managing OSAHS over a period of 14 years with and without CPAP from the perspective of the UK's National Health Service (NHS)

\section{Data sources}

A systematic literature search was performed using a search term of "obstructive sleep apnoea" plus one of the following: incidence, prevalence, epidemiology, hypertension, cardiovascular event, myocardial infarction (MI), stroke, cerebrovascular event, depression, diabetes, compliance, road traffic accident (RTA), utilities, quality of life, costeffectiveness, cost-utility, resource utilisation, economic and cost. The search strategy was not limited by year of publication, but only English language papers were included. A manual literature search was also undertaken, based on citations in the published papers.

\section{Economic model}

A Markov model was constructed which depicted the management of a 55-year-old patient with severe OSAHS as defined by an AHI >30 and daytime sleepiness (ESS $\geqslant 12$ ) (fig 1). Within the model, the time to the start of treatment with CPAP following the initial outpatient visit or diagnostic sleep study (whichever comes first) is 8.4 months (based on information supplied by the interviewees) and the model spans a period of 14 years.

The model comprises the following health states and death:

- Event-free with uncontrolled OSAHS.

- Event-free with controlled OSAHS

- Stroke.

- Cardiovascular event.

- RTA.

- Survival following stroke, cardiovascular event or RTA.

Patients can initially move into one of four health states (i.e. event-free, stroke, cardiovascular event or RTA) and patients remain in a state for 


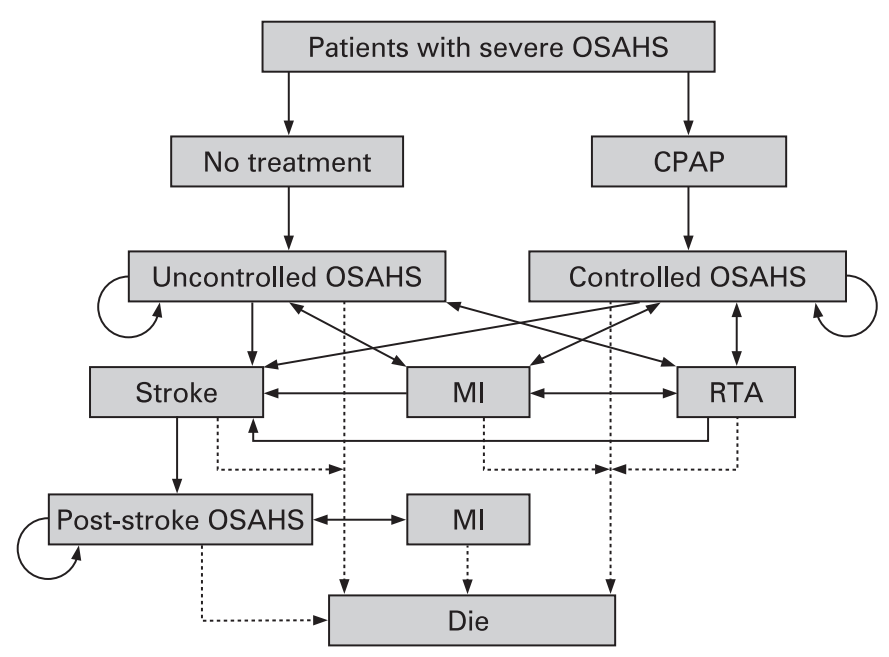

Figure 1 Markov model depicting the management of severe obstructive sleep apnoea/hypopnoea syndrome (OSAHS). CPAP, continuous positive airway pressure; $\mathrm{MI}$, myocardial infarction; RTA, road traffic accident.

1 year at a time before moving to another state. Patients who have a cardiovascular event or RTA in any one year can have a stroke or another cardiovascular event or RTA in a later year, and there are no limits on the number of events they can undergo in subsequent years. However, patients who have a stroke can no longer drive, but there are no limits on the number of cardiovascular events or strokes they can undergo in subsequent years. One limitation of the model structure is that it does not consider the impact of patients with OSAHS having an RTA as a result of being a passenger, particularly patients with stroke who can no longer drive. The arrows depict the possible movement of patients between the different health states (fig 1).

\section{Model inputs: clinical \\ Cardiovascular events}

After sleepiness, the principal morbidity of OSAHS relates to the cardiovascular system. ${ }^{3}$ However, identification of a clear association between OSAHS and cardiovascular disease has proved challenging, ${ }^{3}$ although epidemiological studies have consistently found an association between OSAHS and hypertension. ${ }^{4-6}$

A recently published observational (non-randomised) study by Marin et $a l^{7}$ compared the cumulative risk of fatal and nonfatal cardiovascular and cerebrovascular episodes in patients with OSAHS over 12 successive years among those who received and those who declined CPAP. The study found that patients with severe disease (AHI >30) who were treated with CPAP had a significantly lower risk of cardiovascular events than untreated patients. However, this was an uncontrolled study, and patients who decline medical advice and treatments have been shown to have a higher mortality independent of the treatment itself. ${ }^{8}$ This difference in cardiovascular risk may therefore be an overestimate.

The cumulative annual risk of fatal and non-fatal cardiovascular and cerebrovascular events in patients with severe disease treated with and without CPAP in the study by Marin et al ${ }^{7}$ was extrapolated using a best fit exponential model from 12 to 14 years, from which the annual incidence was calculated. In a separate study, Mar et $a l^{9}$ found that the risk of coronary heart disease and stroke in patients with untreated severe OSAHS was 1.185 and 1.353 , respectively. Hence, the ratio of developing coronary heart disease to stroke was 1:1.13. In treated patients the risk was 1:1. These ratios were applied to our estimates of the annual incidence of fatal and non-fatal cardiovascular and cerebrovascular events in patients with severe OSAHS, enabling the annual risk of cardiovascular event, stroke, fatality and nonfatality from a cardiovascular and cerebrovascular event to be estimated. These estimates were incorporated into the Markov model and represent the likely maximum effect.

\section{Road traffic accidents}

Only two studies were found in which the relative risk of RTAs in patients with OSAHS compared with control populations was reported. The first was a case-control study ${ }^{10}$ which found that the risk of an RTA in untreated patients was three times greater than the risk in control populations, while the risk in patients treated with CPAP was the same as that of control populations. In a second study, ${ }^{11}$ the number of RTAs in patients with OSAHS was estimated using a road safety platform. The authors estimated that the risk of an RTA in untreated patients was 2.3 times greater than the risk among control populations, whereas the risk in treated patients was marginally less than that of control populations. By taking the average of the findings of these two studies, ${ }^{10}{ }^{11}$ the model assumes that the risk of an RTA in untreated patients was 2.6 times greater than the risk in control populations, whereas the risk in treated patients was the same as that of control populations.

According to the Department of Transport, ${ }^{12}$ there were 221751 RTAs in Great Britain in 2002 of which 1.3\% resulted in a fatality. The distribution of different severities of RTAs (slight, serious and fatal) in Great Britain in 2002 and corresponding costs ${ }^{12}$ have been incorporated into the model. In the same year there were 25.3 million licensed motor vehicles in Great Britain. Hence, by assuming that all patients are drivers and have a licensed motor vehicle, it was estimated that the risk of an RTA in the control population (and thus in treated patients with OSAHS) is 0.009 per annum, and 0.023 per annum in untreated patients with OSAHS.

\section{Compliance}

Only four published studies were found which reported the percentage of patients with OSAHS who continued using their fixed pressure CPAP device over various periods: $68 \%$ over 60 months, ${ }^{13} 85 \%$ over 84 months, ${ }^{14} 72 \%$ over 24 months ${ }^{15}$ and $80 \%$ over an unspecified period. ${ }^{16}$ The average of these four studies was estimated to be $74 \%$. McArdle et $a l^{13}$ also reported that the percentage of patients who continued using their CPAP device fell from $84 \%$ at the end of the first year to $68 \%$ after 4 years, remaining at this level for a further 3 years. This equates to a discontinuation rate of $5 \%$ per annum over 4 years. Similarly, Krieger et al ${ }^{14}$ reported that the percentage of patients who continued using their CPAP device fell from $90 \%$ after 3 years to $85 \%$ after 7 years, equivalent to a discontinuation rate of $1 \%$ per annum over 4 years. The weighted average of these two discontinuation rates was estimated to be $3.8 \%$ per annum.

Since the period of the model is $>1$ year, it was assumed that $74 \%$ of all patients would continue using their device during the first year of treatment. It was also assumed that $3.8 \%$ of patients receiving CPAP at the beginning of the second year would discontinue using the device during the year, and this 
Table 1 Estimates of healthcare resource use (95\% confidence intervals in parentheses)

\begin{tabular}{ll}
\hline Resource & Probability \\
\hline Probability of having an initial outpatient visit before a diagnostic sleep study & 0.31 (0.11 to 0.51) \\
Probability of one outpatient visit after a diagnostic sleep study & $0.69(0.49$ to 0.89$)$ \\
Probability having of a home sleep study & $0.75(0.59$ to 0.90$)$ \\
Probability of having a home titration study & $0.99(0.97$ to 1.00$)$ \\
Probability of having a titration study in hospital & $0.04(0$ to 0.05$)$ \\
Probability of using CPAP (fixed) for titration & $0.19(0.01$ to 0.36$)$ \\
Probability of using CPAP (auto) for titration & $0.81(0.64$ to 0.99$)$ \\
Probability of seeing a consultant during the titration phase & $0.40(0.05$ to 0.52$)$ \\
Probability of seeing a specialist nurse during the titration phase & $1.00(0.53$ to 1.00$)$ \\
Probability of seeing a technician during the titration phase & $0.48(0.10$ to 0.93) \\
Probability of having a humidifier & $0.38(0.22$ to 0.50$)$ \\
Probability of switching from fixed to auto CPAP in the second year & $0.06(0.04$ to 0.07$)$ \\
Probability of switching from fixed to auto CPAP in subsequent years & $0.01(0$ to 0.02$)$ \\
Probability of a non-compliant patient returning their machine & $0.75(0.50$ to 1.00$)$ \\
Probability of having a follow-up visit within 3 months of starting CPAP & $0.75(0.50$ to 1.00$)$ \\
Probability of having a follow-up visit within 4-6 months of starting CPAP & $0.75(0.75$ to 1.00) \\
Probability of annual follow-up visits after starting CPAP with a consultant & $0.13(0$ to 0.27$)$ \\
Probability of annual follow-up visits after starting CPAP with a specialist nurse & $0.61(0.33$ to 0.79) \\
Probability of annual follow-up visits after starting CPAP with a technician & $0.26(0.09$ to 0.54) \\
Probability of a deceased patient's machine being returned & $0.90(0.75$ to 1.00$)$ \\
\hline
\end{tabular}

CPAP, continuous positive airway pressure.

discontinuation rate would decline exponentially over the remaining period of the model.

\section{Model inputs: resource use}

No publications were identified that quantified healthcare resource use for the management of OSAHS in the UK. This was therefore estimated using information obtained from interviews with 19 randomly selected clinicians from across the UK who managed large established sleep services and who collectively see $>6000$ new patients with OSAHS per annum. The use of healthcare resources attributable to managing patients with severe OSAHS that has been modelled is summarised in table 1. According to these clinicians, the shelf life of a CPAP device within their individual hospitals was 7 years, and this was the time frame adopted by the National Institute for Health and Clinical Excellence (NICE) in their health technology appraisal of CPAP. ${ }^{17}$

Unit costs at 2005/6 prices incorporated into the model were obtained from the Departments of Health and Transport. ${ }^{12} 18$ The costs of CPAP, humidifier and mask used in the model are the list prices without the application of any discounts often enjoyed by the NHS (ResMed, Oxfordshire, UK, 2007). The hospital cost of an episode of myocardial infarction ${ }^{19}$ and the cost of home-based cardiac rehabilitation for the first year following a myocardial infarction ${ }^{20}$ have been used as proxies for the cost of managing a cardiovascular event.

Model inputs: health-related quality of life (HROoL) and utilities A Spanish study obtained utility values for different health states from patients with OSAHS who completed the EQ-5D questionnaire before and after they received CPAP treatment. ${ }^{9}$ In the absence of any other data, these utilities (0.738 for untreated OSAHS, 0.811 for treated OSAHS, 0.590 for non-fatal stroke in untreated OSAHS patients, 0.649 for non-fatal stroke in treated OSAHS patients, 0.664 for non-fatal cardiovascular event in untreated OSAHS patients, 0.730 for non-fatal cardiovascular event in treated OSAHS patients, 0.701 (estimated) for non-fatal RTA in untreated OSAHS patients and 0.771 (estimated) for non-fatal RTA in treated OSAHS patients) were applied to the health states in our Markov model, enabling the level of health gain in terms of the expected number of quality-adjusted life years (OALYs) over 14 years to be estimated.

\section{Model outputs}

The measures of clinical effectiveness were defined as:

- The expected percentage of surviving patients at 14 years.

- The expected percentage of event-free surviving patients at 14 years.

- The expected number of OALYs at 14 years.

The model also estimated the cumulative risk of having a stroke, cardiovascular event and RTA over 14 years. By incorporating the unit costs into the different states within the Markov model, the expected direct healthcare costs over 14 years were estimated. Costs and consequences that were incurred by patients in the second and subsequent years of the model were discounted by $3.5 \%$ in accordance with current UK guidelines. ${ }^{21}$

\section{Cost-effectiveness analyses}

The incremental cost-effectiveness of CPAP relative to no treatment was calculated as the difference between the expected cost of the two strategies over 14 years divided by

Table 2 Expected outcomes associated with severe OSAHS after 14 years of treatment with CPAP or no treatment

\begin{tabular}{|c|c|c|}
\hline \multirow[b]{2}{*}{ Outcome } & \multicolumn{2}{|c|}{ Probability after 14 years following: } \\
\hline & No treatment & CPAP \\
\hline Survival & $0.57(0.49$ to 0.65$)$ & $0.72(0.66$ to 0.78$)$ \\
\hline Cardiovascular event & $0.35(0.20$ to 0.53$)$ & $0.19(0.09$ to 0.27$)$ \\
\hline Stroke & $0.39(0.23$ to 0.60$)$ & $0.20(0.08$ to 0.31$)$ \\
\hline RTA & $0.24(0.21$ to 0.28$)$ & $0.17(0.14$ to 0.19$)$ \\
\hline Event-free survival & $0.30(0.13$ to 0.46$)$ & $0.58(0.47$ to 0.70$)$ \\
\hline QALYs & 7.22 (6.48 to 7.93$)$ & $8.09(7.17$ to 8.44$)$ \\
\hline
\end{tabular}

$95 \%$ confidence intervals are shown in parentheses.

CPAP, continuous positive airway pressure; OSAHS, obstructive sleep apnoea/ hypopnoea syndrome; RTA, road traffic accident; $Q A L Y$, quality-adjusted life year. 
Table 3 Expected healthcare costs (at 2005/6 prices) over 14 years following no treatment or CPAP

\begin{tabular}{lcc}
\hline & \multicolumn{2}{c}{$\begin{array}{l}\text { Expected discounted NHS costs over 14 } \\
\text { years at 2005/6 prices following: }\end{array}$} \\
\cline { 2 - 3 } Resource & No treatment (f) & CPAP (f) \\
\hline Clinician visits for OSAHS & $0.00(0)$ & $682.22(7)$ \\
Devices & $0.00(0)$ & $1794.52(19)$ \\
Diagnostic sleep studies & $0.00(0)$ & $123.60(1)$ \\
Resources required to manage & $1044.67(10)$ & $564.50(6)$ \\
cardiovascular events & & \\
Resources required to manage strokes & $7203.58(68)$ & $4961.12(51)$ \\
Resources required to manage RTAs & $2396.77(23)$ & $1546.29(16)$ \\
Total & $10645.02(100)$ & $9672.25(100)$ \\
\hline
\end{tabular}

Percentage of total expected cost is shown in parentheses.

CPAP, continuous positive airway pressure; NHS, National Health Service;

OSAHS, obstructive sleep apnoea/hypopnoea syndrome; RTA, road traffic accident.

the difference between the expected outcomes between the two strategies over 14 years. When the outcome was the probability of survival, the incremental cost-effectiveness of CPAP relative to no treatment was defined as the cost per life-year gained. When the outcome was the expected number of OALYs, the incremental cost-effectiveness of CPAP relative to no treatment was defined as the cost per OALY gained. If a treatment resulted in improved outcome for less cost, it was defined as a "dominant treatment".

\section{Sensitivity analyses}

Probabilistic sensitivity analyses were undertaken by simultaneously varying all the probabilities, utilities, unit costs and resource use values within the model. The probabilities and utilities were varied randomly according to a beta distribution and the resource use estimates and unit costs were varied randomly according to a normal distribution. Deterministic analyses were performed to assess the impact of varying individual parameters within the model.

\section{RESULTS}

\section{Expected outcomes of care}

According to the Markov model, $57 \%$ of untreated patients are expected to be alive at the end of 14 years compared with $72 \%$ of patients treated with CPAP. Moreover, $30 \%$ of untreated patients are expected to have survived event-free over 14 years compared with $58 \%$ of those treated with CPAP. The expected changes in outcomes associated with CPAP over 14 years are summarised in table 2 .

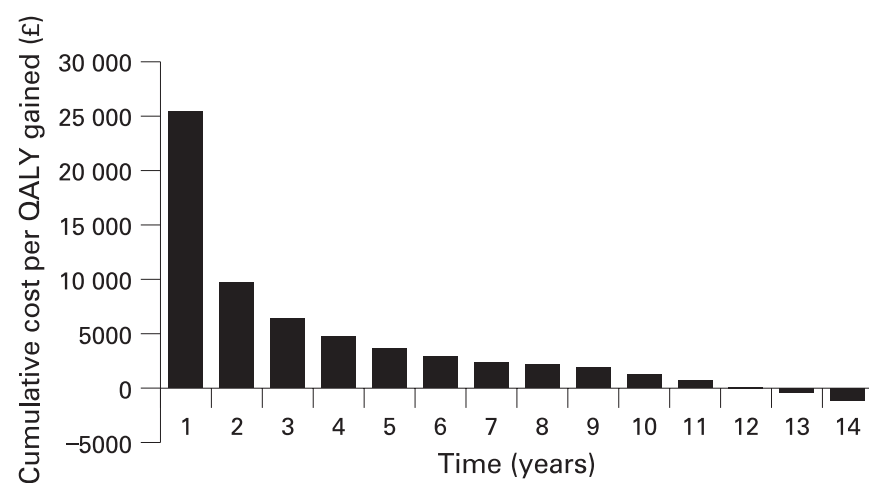

Figure 2 Cumulative cost per quality-adjusted life year (OALY) gained over 14 years.

\section{Expected healthcare costs}

According to the Markov model, untreated patients are expected to cost the NHS $£ 10645$ (95\% confidence interval (CI) $£ 7988$ to $£ 14$ 098) per patient over 14 years compared with $£ 9672$ (95\% CI $£ 8057$ to $£ 12$ 860) per CPAP-treated patient (table 3). For a cost reduction of $£ 973$ (95\% CI - £1983 to £1508) over 14 years, the use of CPAP over 14 years is therefore expected to:

- increase the probability of survival by $25 \%$;

- decrease the relative risk of having a cardiovascular event by $46 \%$;

- decrease the relative risk of having a stroke by $49 \%$;

- decrease the relative risk of having an RTA by $31 \%$;

- increase the probability of event-free survival by $92 \%$.

The total discounted cost for the device, mask, humidifier and sundries was estimated to be $£ 1795$ per patient over 14 years. This cost reflects an expectation that the CPAP device would be returned in $75 \%$ and $90 \%$ of cases where patients either did not comply or died, respectively.

The cost associated with managing stroke was found to be the primary cost driver in both groups. The secondary cost driver in untreated patients was found to be the cost associated with managing RTAs, whereas in CPAP-treated patients it was the cost of the device itself.

The cumulative NHS costs associated with the management of OSAHS accrue over time. The cost of managing patients with CPAP becomes less than that of patients not receiving CPAP treatment by the 13th year owing to the greater number of cardiovascular events, strokes and RTAs in untreated patients.

\section{Cost-effectiveness analyses}

The cost-effectiveness analyses of CPAP relative to no treatment show that CPAP affords the NHS a dominant treatment. However, fig 2 illustrates that treatment of CPAP for a period of 1 year is not a cost-effective option since the cost per OALY gained is $>£ 20000$. However, after 2 years of treatment the cost per OALY gained is $£ 10000$ or less and, after 13 years of treatment, CPAP becomes a dominant treatment.

\section{Sensitivity analyses}

Probabilistic sensitivity analyses showed that CPAP has a 0.99 probability of being cost-effective for a threshold of $£ 20000$ per OALY.

The model incorporated clinical outcomes pertaining to cardiovascular and cerebrovascular events and RTAs from disparate publications. The base case analyses were repeated by removing these outcomes from the model. Even if all cardiovascular and cerebrovascular events and RTAs were excluded from the model, the cost per OALY gained with CPAP relative to no treatment would be $£ 4592$ (95\% CI -£1236 to £5112). Hence, CPAP still affords the NHS a cost-effective technology.

Deterministic sensitivity analyses revealed that changes in some input values had an effect on the relative cost-effectiveness of CPAP, particularly the proportion of patients who continue using CPAP, the risk of having a cardiovascular/ cerebrovascular event, the risk of having an RTA, the utility for treated and untreated OSAHS, the cost of managing a non-fatal RTA and the cost of managing stroke rehabilitation. Nevertheless, CPAP ceases to afford the NHS a dominant treatment only when:

- the proportion of patients who continue using CPAP falls below $60 \%$ in the first year of treatment; 
- the relative risk of having a cardiovascular/cerebrovascular event falls to $60 \%$ below the base case value;

- the cardiovascular event:stroke ratio among untreated OSAHS patients rises above 1:0.9.

However, in all instances the cost per OALY gained with CPAP is $<£ 5000$. The model was insensitive to changes in other model inputs. In particular, the cost-effectiveness of CPAP only changed marginally when changes were made to (1) the time to the start of treatment and (2) all the utility values except that for untreated OSAHS.

The base-case model assumes that the shelf life of CPAP within the NHS is 7 years. However, changing the shelf life to 5 years has a negligible effect on the cost-effectiveness of CPAP. The expected cost to the NHS would rise by $£ 160$ to $£ 9833$ (95\% CI $£ 8229$ to $£ 13060$ ) per patient over 14 years. Nevertheless, CPAP is still expected to afford the NHS a dominant treatment with a cost per OALY of $-£ 942$ (95\% CI $-£ 2507$ to $£ 4173)$.

\section{DISCUSSION}

The model was constructed using information obtained from disparate publications, including the incidence of cardiovascular and cerebrovascular events reported by Marin et $a$ in their uncontrolled observational study. ${ }^{7}$ The rationale for using this particular study was that patients were followed for 12 years, which was the longest study we could find. Such observational studies have inherent weaknesses such as a placebo effect, a Hawthorne effect and regression to the mean. Notwithstanding this, the findings of Marin et al were concordant with those of Peker et al. ${ }^{22}$ Published randomised trials have reported the effect of CPAP on blood pressure. Accordingly, we could have used Framingham risk equations to provide a link between blood pressure and the incidence of fatal and non-fatal cardiovascular events in a hypothetical cohort of patients. However, we considered that using clinical outcomes from a cohort of patients with OSAHS based on actual clinical practice would lead to fewer assumptions and a model that was more representative of the "real world". Additionally, the analysis was unable to consider the impact of other co-morbidities, such as diabetes and depression, due to a lack of published evidence.

Our model is subject to several other limitations. Since the clinical basis of the model was disparate published studies and Government statistics, the model may not reflect clinical outcomes observed in clinical practice among a cohort of patients over a period of successive years.

Moreover, it was not possible to model the management of moderate OSAHS owing to the lack of published clinical data required to inform such modelling. We were unable to find any published studies assessing healthcare resource utilisation associated with managing OSAHS in the UK. Hence, this was obtained from interviews with 19 clinicians from across the UK who collectively see $>6000$ new patients with OSAHS per annum. Utilities were derived from a Spanish population, and these may not be the same as those of a UK population. This gives rise to uncertainty regarding the applicability of these values to a UK study, although sensitivity analyses showed that changing most of these values had minimal impact on the costeffectiveness of CPAP.

The model used resource estimates and utility values for the "average patient" and did not take into account such factors as age, sex, suitability of patients for different treatments, other disease-related factors, patients' preferences and level of clinicians' skills.

The model only considered direct healthcare costs borne by the NHS and not those borne by other Government departments, such as social services costs for rehabilitating patients with stroke and the wider societal costs of RTAs (eg, a fatal RTA can cost $>£ 1$ million $^{23}$ ). Moreover, the model excluded some primary care costs such as those associated with managing sleepiness, RTAs and cardiovascular events, and this may have underestimated the health economic benefits of CPAP. Also excluded is the cost of any medication including that of antihypertensive drugs required to manage hypertension arising from OSAHS. The model also excluded costs incurred by patients and indirect costs borne by patients or society including the societal cost of sleepiness. If all these costs were factored in, the health economic benefits of CPAP therapy would inevitably be much greater.

Notwithstanding these limitations, our model indicated that treatment with CPAP for a period of 1 year is not a costeffective option since the cost per OALY gained is $>£ 20000$. However, NICE considers that a technology that has a costeffectiveness of $<£ 20000$ per OALY potentially affords an effective use of NHS resources. ${ }^{21}$ The use of CPAP over 14 years is therefore expected to afford the NHS a cost-effective technology since, after 2 years of treatment with CPAP, the cost per OALY gained is $<£ 10000$ and, after 13 years of treatment, CPAP becomes dominant. These findings are concordant with those from four other studies, all of which found CPAP to be a cost-effective treatment. ${ }^{94-26}$ Sensitivity analyses showed that the relative cost-effectiveness of CPAP is sensitive to continuation rates. Thus, any intervention that improves long-term take-up rates such as better patient education is worth employing.

In conclusion, within the limitations of our model, CPAP was found to be clinically more effective than no treatment and, from the perspective of the NHS in the UK, a cost-effective strategy after a minimum of 2 years of treatment.

Acknowledgements: The authors thank the following clinicians for their contributions: Dr D Banerjee, Birmingham Heartlands, Birmingham; Dr B Cooper, Queen Elizabeth Hospital, Birmingham; Dr A Cummin, Charing Cross Hospital, London; Dr C Davies, Royal Berkshire Hospital, Reading; Dr C Dodds, South Cleveland Hospital, Middlesbrough; Dr M Elliott, St James University Hospital, Leeds; Dr P Fitch, Aberdeen Royal Infirmary, Aberdeen; Professor J Gibson, Freeman Hospital, Newcastle upon Tyne; Dr C Gelder, Llandough Hospital, Cardiff; Dr M A Greenstone, Castle Hill Hospital, Hull; Dr C Hanning, Leicester General Hospital, Leicester; Dr A Kendrick, Bristol General Hospital, Bristol; Dr T MacKay, Royal Infirmary of Edinburgh, Edinburgh; Dr J Pepperall, Musgrove Park Hospital, Taunton; Dr M Polkey, Royal Brompton Hospital, London; Dr R Royston, Royal National Throat, Nose and Ear Hospital, London; Dr J White, York District General Hospital, York; Dr A Williams, St Thomas' Hospital, London.

Funding: This study was sponsored financially by ResMed, Oxfordshire, UK, manufacturers of CPAP S8 Escape and APAP S8 AutoSet Spirit.

Competing interests: None.

\section{REFERENCES}

1. Smith I, Lasserson TJ, Wright J. Drug therapy for obstructive sleep apnoea in adults Cochrane Database Syst Rev 2006;(3).

2. Giles TL, Lasserson TJ, Smith BJ, et al. Continuous positive airways pressure for obstructive sleep apnea in adults. Cochrane Database Syst Rev 2006; (3)

3. McNicholas WT, Krieger $\mathrm{J}$ on behalf of the ERS Task Force. Public health and medicolegal implications of sleep apnoea. Eur Respir $J$ 2002;20:1594-609.

4. Duran J, Esnaola S, Rubio R, et al. Obstructive sleep apnea-hypopnea and related clinical features in a population based sample of subjects aged 30 to $70 \mathrm{yr}$. Am J Respir Crit Care Med 2001;163:608-13.

5. Iellamo F, Montano N. Continuous positive airway pressure treatment: good for obstructive sleep apnea, maybe not for hypertension? Chest 2006;129:1403-4.

6. Campos-Rodriguez F, Grilo-Reina A, Perez-Rochel J, et al. Effect of continuous positive airway pressure on ambulatory BP in patients with sleep apnea and hypertension. Chest 2006;129:1459-67. 
7. Marin J, Carrizo SJ, Vicente E, et al. Long-term cardiovascular outcomes in men with obstructive sleep apnoea-hypopnoea with or without treatment with continuous positive airway pressure: an observational study. Lancet 2005:365:1046-53.

8. Granger BB, Swedberg K, Ekman I, et al. Adherence to candesartan and placebo and outcomes in chronic heart failure in the $\mathrm{CH} / \mathrm{ARM}$ programme: double-blind, randomised, controlled clinical trial. Lancet 2005;366:2005-11.

9. Mar J, Rueda JR, Duran-Cantolla J, et al. The cost-effectiveness of nCPAP treatment in patients with moderate-to-severe obstructive sleep apnoea. Eur Respir J 2003;21:515-22.

10. George GF. Reduction in motor vehicle collisions following treatment of sleep apnoea with nasal CPAP. Thorax 2001;56:508-12.

11. Mazza S, Pepin J-L, Naegele B, et al. Driving ability in sleep apnoea patients before and after CPAP treatment: evaluation on a road safety platform. Eur Respir $J$ 2006;28:1020-8.

12. Department of Transport. Highways economic note 1. London: Department of Transport, 2004.

13. McArdle N, Devereux G, Heidarnejad H, et al. Long-term use of CPAP therapy for sleep apnea/hypopnea syndrome. Am J Respir Crit Care Med 1999;159/4 Pt 1):1108-14.

14. Krieger J, Kurtz D, Petiau C, et al. Long-term compliance with CPAP therapy in obstructive sleep apnea patients and in snorers. Sleep 1996;19(9 Suppl):S136-43

15. Findley LJ, Smith C, Hooper J, et al. Treatment with nasal CPAP decreases automobile accidents in patients with sleep apnea. Am J Respir Crit Care Med 2000;161:857-9

16. Marquez-Baez C, Paniagua-Soto J, Castilla-Garrido JM. Treatment of sleep apnea syndrome with CPAP: compliance with treatment, its efficacy and secondary effects. Rev Neurol 1998;26:375-80.
17. National Institute for Health and Clinical Excellence (NICE). Continuous positive airway pressure for the treatment of obstructive sleep apnoea/hypopnoea syndrome. NICE Technology Appraisal Guidence 139. London: NICE, 2003.

18. Department of Health. Reducing brain damage: faster access to better stroke care. London: National Audit Office, 2005.

19. Department of Health. NHS reference costs 2005/06. London: Department of Health, 2006. http://www.dh.gov.uk/PublicationsAndStatistics/Publications/ PublicationsPolicyAndGuidance/PublicationsPolicyAndGuidanceArticle/fs/ en?CONTENT ID = 4141135\&chk = 7wrsFg.

20. Taylor RS, Watt A, Dalal HM, et al. Home-based cardiac rehabilitation versus hospital-based rehabilitation: a cost effectiveness analysis. Int J Cardiol 2007:119:196-201.

21. National Institute for Health and Clinical Excellence (NICE). Guide to the methods of technology appraisal. Reference N0515. London: NICE, 2004. http:// www. nice.org.uk/niceMedia/pdf/TAP Methods.pdf.

22. Peker Y, Carlson J, Hedner J. Increased incidence of coronary artery disease in sleep apnoea: a long-term follow-up. Eur Respir J 2006;28:596-602.

23. Douglas NJ, George CFP. Treating sleep apnoea is cost effective. Thorax 2002;57:93-4.

24. Albarrak M, Banno K, Sabbagh AA, et al. Utilization of healthcare resources in obstructive sleep apnea syndrome: a 5-year follow-up study in men using CPAP. Sleep 2005;28:1306-11.

25. Tousignant $\mathbf{P}$, Cosio MG, Levy RD, et al. Quality adjusted life years added by treatment of obstructive sleep apnea. Sleep 1994;17:52-60.

26. Working Group on Acute Purchasing. Nasal continuous positive airways pressure in the management of sleep apnoea. Note for Purchasers: 00/06. Trent Institute for Health Services Research, Universities of Leicester, Nottingham and Sheffield, October 2000.

\section{Lung alert}

\section{When is it too EARLY to start bosentan in pulmonary arterial hypertension?}

Pulmonary arterial hypertension is a debilitating progressive disease which eventually leads to right heart failure and death. Although observational studies indicate that early treatment initiation might be advantageous, previous clinical trials only looked at the use of bosentan (dual endothelin receptor antagonist) in patients with advanced symptomatic states (WHO functional class III and IV).

The EARLY trial enrolled 185 patients aged $\geqslant 12$ years with mildly symptomatic (WHO functional class II) pulmonary arterial hypertension who were randomised to receive either bosentan at an initial dose of $62.5 \mathrm{mg}$ twice daily, up-titrated to $125 \mathrm{mg}$ twice daily after 4 weeks, or placebo for 6 months. Treatment with other approved agents was prohibited with the exception of sildenafil. The primary end point was improvement in exercise capacity (reflected by 6 min walk distance), a surrogate for cardiopulmonary haemodynamics. Secondary end points included time to clinical worsening and change from baseline functional class. Analysis of the primary end points (in 168 patients) showed a reduction in the mean pulmonary vascular resistance ( $83.2 \%$ of baseline value). The initial increase in exercise capacity also seen in the bosentan group was not statistically significant at 6 months. The overall number of adverse effects was similar between groups, with syncope the most common serious adverse event in the bosentan group.

The study was not sufficiently powered to perform any subgroup analysis. The EARLY study showed the potential benefit of bosentan in mildly symptomatic patients with pulmonary arterial hypertension, as reflected by improvement in haemodynamics and the prevention of clinical deterioration.

- Galie N, Rubin LJ, Hoeper MM, et al. Treatment of patients with mildly symptomatic pulmonary arterial hypertension with bosentan (EARLY study): a double-blind, randomised controlled trial. Lancet 2008;371:2093-100

\section{F Magdy}

Correspondence to: Dr F Magdy, Junior Trainee in Cardiology, University Hospital of North Staffordshire, Stoke-on-Trent, UK; fmfouadissa@yahoo.co.uk 